\title{
Anti-PD-1/Anti-PD-L1 Bispecific Antibody LY3434172
}

National Cancer Institute

\section{Source}

National Cancer Institute. Anti-PD-1/Anti-PD-L1 Bispecific Antibody LY3434172. NCI

Thesaurus. Code C160605.

A bispecific antibody targ eting both the human negative immunoregulatory checkpoint receptor programmed cell death protein 1 (PD-1; PDCD1; CD279) and its ligand, human programmed death-ligand 1 (PD-L1; CD274), with potential checkpoint inhibitory, immunostimulating and antineoplastic activities. Upon administration, anti-PD-1/anti-PDL1 bispecific antibody LY3434172 simultaneously targets and binds to PD-1, which is expressed on a variety of leukocyte subsets including activated T-lymphocytes in the tumor microenvironment (TME), and PD-L1 expressed on tumor cells. This prevents PDL1 from binding to and activating its receptor PD-1 and inhibits the PD-L1/PD-1-mediated downregulation of T-cell activation and proliferation. This abrogates T-cell inhibition, activates antigen-specific T-lymphocytes and enhances cytotoxic T-cell-mediated tumor cell lysis, which may lead to a reduction in tumor growth. PD-L1 binding to PD-1 on activated T-cells inhibits the expansion and survival of CD8-positive T-cells, suppresses the immune system and results in immune evasion. 